\title{
NOTE ON SOME GAP THEOREMS
}

\section{DIETER GAIER}

Many theorems in the theory of series concern gap series of the form

$$
\begin{array}{r}
\sum_{m=0}^{\infty} a_{m} \quad \text { with } a_{m}=0 \text { for } m_{k}<m \leqq M_{k} \text { and } m_{k} \nearrow \infty, \\
\text { where } M_{k} \geqq m_{k}(1+\vartheta) \text { for some } \vartheta>0,
\end{array}
$$

with partial sums $s_{m}$. These theorems infer the convergence of the sequence $\left\{s_{m_{k}}\right\}$ for $k \rightarrow \infty$ from assumptions concerning the summability of (1) or from properties of the associated complex function

$$
f(z)=\sum_{m=0}^{\infty} a_{m} z^{m} \quad(z=x+i y) .
$$

Two theorems of the latter type are the following Theorems $\mathrm{A}$ and B. ${ }^{1}$

Theorem A [1]. Suppose that a series (1) is given and that (2) is regular in $|z|<1$ and continuous in a circle $|z-\alpha| \leqq 1-\alpha$ for some $\alpha$ with $0<\alpha<1$. Then $s_{m_{k}} \rightarrow f(1)(k \rightarrow \infty) .^{2}$

Theorem B [4]. Suppose that a series (1) is given and that (2) is regular in $|z|<1$ and bounded in a sector $|\arg z|<\epsilon, 0<|z|<1$. Then $\lim _{x \rightarrow 1-0} f(x)=s$ implies $s_{m_{k}} \rightarrow s(k \rightarrow \infty)$.

We are going to prove a theorem which contains both of these theorems:

THEOREM 1. Suppose that a series (1) is given and that (2) is regular in $|z|<1$ and bounded in a circle $|z-\alpha|<1-\alpha$ for some $\alpha$ with $0<\alpha<1$. Then $\lim _{x \rightarrow 1-0} f(x)=s$ implies $s_{m_{k}} \rightarrow s(k \rightarrow \infty)$.

By a short complex variable argument, we shall reduce the proof of Theorem 1 to the following gap theorem on summable series of type (1).

Theorem 2. Suppose that a series (1) is given and that

Received by the editors February 1, 1956 and, in revised form, March 19, 1956.

${ }^{1}$ In this connection, see also some gap theorems by Noble $[8 ; 9]$.

2 Evgrafov has also given an example to show that Theorem A may be false if $|z-\alpha| \leqq 1-\alpha$ is replaced by a sector $\pi / 2+\delta \leqq \arg (z-1) \leqq 3 \pi / 2-\delta, 0 \leqq|z-1| \leqq \rho$ $(\delta>0, \rho>0)$. 
(i) the function (2) is regular in $|z|<\alpha$ and at $z=\alpha$ for some $\alpha$ with $0<\alpha<1$;

(ii) at the point $z=1$, the Taylor series of $f(z)$ about $z=\alpha$ is $C_{1^{-}}$summable to the value s.

Then there exists a number $\delta=\delta(\vartheta, \alpha)>0$ such that under the additional hypothesis

$$
s_{m}=O\left((1+\delta)^{m}\right) \quad(m \rightarrow \infty)
$$

one can conclude

$$
s_{m_{k}} \rightarrow s \quad(k \rightarrow \infty) .
$$

REMARKs. (a) An equivalent form of (ii) is

(ii') the series (1) is $C_{1} T_{\alpha}$-summable to the value $s$, where $T_{\alpha}$ denotes the "circle method" of order $\alpha .^{3}$

(b) Our proof of Theorem 2 goes beyond the theorem; it establishes the following extension:

The conclusion (3) in Theorem 2 remains valid if $C_{1}$-summability in (ii) or (ii') is replaced by $C_{x}$-summability; here $\kappa$ can be any number $\geqq 0$.

For $\kappa=0$ a similar theorem, with $B$ (Borel) instead of $T_{\alpha}$, has been proved by Zygmund (see [5, p. 206]); our theorem or its extension can, like Zygmund's theorem, be used for a proof of Ostrowski's theorem on overconvergence. Theorems similar to Theorem 2 and its extension could also be obtained by our method of proof for $B$ (Borel) and $E_{p}$ (Euler-Knopp) instead of $T_{\alpha}$.

The author wishes to thank the referee for a number of suggestions.

Proof of Theorem 1. We show that all conditions of Theorem 2 are satisfied. Consider the development $\sum_{m=0}^{\infty} a_{m}^{\prime}(z-\alpha)^{m}$ of $f(z)$ at $z=\alpha$. Since $f(z)$ is bounded for $|z-\alpha|<1-\alpha$, the Abel-summability of $\sum_{m=0}^{\infty} a_{m}^{\prime}(1-\alpha)^{m}$ to $s$ implies the $C_{1}$-summability of the series to the same value (see for example [2, p. 327]). The condition (iii) of Theorem 2 is also satisfied for every $\delta>0$; hence Theorem 2 is applicable and it is sufficient to prove Theorem 2 .

Proof of Theorem 2. We shall use our hypotheses in the form (i), (ii'), (iii). Let $\left\{s_{n}^{\prime}\right\}$ be the $T_{\alpha}$-transform of $\left\{s_{m}\right\}$, i.e.

$$
s_{n}^{\prime}=(1-\alpha)^{n+1} \sum_{m=n}^{\infty}\left(\begin{array}{l}
m \\
n
\end{array}\right) \alpha^{m-n} s_{m}=\sum_{m \geqq n} u_{m}(n) s_{m},
$$

with the notation of $[5$, p. 201] and $k=1-\alpha$, so that, for each fixed value $n$, the maximum term $u_{m}(n)$ is attained for $m=[n /(1-\alpha)]$. Our idea is $201]$.

${ }^{3}$ To obtain the matrix for the "circle method" of order $\alpha$, put $k=1-\alpha$ in $[5, p$. 
(a) to construct for each interval $\left(m_{k}, M_{k}\right)$ in which $\left\{s_{m}\right\}$ is constant $=s_{m_{k}}$, a corresponding interval $\left(n_{k}, N_{k}\right)$ in which $\left\{s_{n}^{\prime}\right\}$ is almost constant and almost $=s_{m_{k}}$ (see equation (12)); $;^{4}$

(b) to deduce from the $C_{1}$-summability of $\left\{s_{n}^{\prime}\right\}$ the convergence of $\left\{s_{n_{k}}^{\prime}\right\}$ and thus the convergence of $\left\{s_{m_{k}}\right\}$ for $k \rightarrow \infty$.

In order to attack (a) for a given $k=1,2, \cdots$, put $\vartheta^{\prime}=\vartheta / 16$ and consider the intervals $I_{1}^{(k)}=\left(m_{k},\left[m_{k}\left(1+\vartheta^{\prime}\right)\right]\right), \cdots, I_{16}^{(k)}=\left(\left[m_{k}(1\right.\right.$ $\left.\left.\left.+15 \vartheta^{\prime}\right)\right],\left[m_{k}\left(1+16 \vartheta^{\prime}\right)\right]\right)$.

For each $k$, let $n_{k}$ be the first index $n$ for which $[n /(1-\alpha)]$ is in $I_{2}^{(k)}$; such an index exists for all sufficiently large $k$. Similarly, let $N_{k}$ be the last index $n$ such that $[n /(1-\alpha)]$ is in $I_{15}^{(k)}$; again, such an index exists for large $k$. Hence we have obtained our intervals $\left(n_{k}, N_{k}\right)$, and we note that by construction

and

$$
\left[\frac{N_{k}}{1-\alpha}\right] \geqq\left[m_{k}\left(1+14 \vartheta^{\prime}\right)\right]
$$

so that

$$
\left[m_{k}\left(1+\vartheta^{\prime}\right)\right] \leqq\left[\frac{n_{k}}{1-\alpha}\right] \leqq\left[m_{k}\left(1+2 \vartheta^{\prime}\right)\right]
$$

$$
N_{k}-n_{k} \geqq \kappa n_{k} \text { for every fixed positive } \kappa<12 \vartheta^{\prime} /\left(1+2 \vartheta^{\prime}\right) \text {. }
$$

Consider now for any $n$ in the interval $\left(n_{k}, N_{k}\right)$ the $T_{\alpha}$-transform (4) and choose a positive number $\sigma<\vartheta^{\prime} /(1-\alpha)(1+\vartheta)$. For each such $n$ the coefficients $a_{m}$ in the interval $[n /(1-\alpha)]-\sigma n \leqq m \leqq[n /(1-\alpha)]$ $+\sigma n$ vanish, for this implies $m_{k}<m \leqq M_{k}$. Therefore, putting $m=[n /(1-\alpha)]+h$, we can estimate

(6) $s_{n}^{\prime}=\sum_{m \geqq n} u_{m}(n) s_{m}=\sum_{|h| \leqq \sigma n} u_{m}(n) s_{m}+\sum_{|h|>\sigma n} u_{m}(n) s_{m}=A_{n}+B_{n}$.

First, let us consider $A_{n}$. We have

$$
\text { (7) } A_{n}=s_{m_{k}} \cdot \sum_{|h| \leqq \sigma n} u_{m}(n)=s_{m_{k}} \cdot\left(1-\sum_{|h|>\sigma n} u_{m}(n)\right)=s_{m_{k}} \cdot\left(1+O\left(e^{-\gamma n}\right)\right)
$$

with some $\gamma=\gamma(1-\alpha, \sigma)>0$ (notation of Theorem 139 in [5]).

Next, we estimate $B_{n}$ with the use of (iii). With a constant $K$ we obtain

$$
\begin{aligned}
\left|B_{n}\right| & \leqq K \sum_{|h|>o n} u_{m}(n)(1+\delta)^{m} \\
& =K(1-\alpha)\left(\frac{1-\alpha}{\alpha}\right)^{n} \sum^{\prime}\left(\begin{array}{l}
m \\
n
\end{array}\right)\{\alpha(1+\delta)\}^{m},
\end{aligned}
$$

4 Incidentally, the assumption (ii) is not needed to prove (12) 
where $\sum^{\prime}$ ranges over all $m$ with $m<[n /(1-\alpha)]-\sigma n$ and $m>[n /(1-\alpha)]+\sigma n$. Put $\alpha^{\prime}=(1+\delta) \alpha$ and assume that $\delta$ in (iii) is so small that

$$
(1+\delta) \alpha<1 \text { and } 0<\frac{1}{1-\alpha^{\prime}}-\frac{1}{1-\alpha}<\frac{\sigma}{2} .
$$

Then we have

$$
\left|B_{n}\right| \leqq K(1-\alpha)\left(\frac{1-\alpha}{\alpha}\right)^{n} \sum^{\prime \prime}\left(\begin{array}{l}
m \\
n
\end{array}\right) \alpha^{\prime m}
$$

where $\sum^{\prime \prime}$ ranges over all $m$ with $m<\left[n /\left(1-\alpha^{\prime}\right)\right]-(\sigma / 2) n$ and $m>\left[n /\left(1-\alpha^{\prime}\right)\right]+(\sigma / 2) n$. This sum we can estimate:

$$
\sum^{\prime \prime}\left(\begin{array}{l}
m \\
n
\end{array}\right) \alpha^{\prime m}=O\left(e^{-\gamma n}\right) \cdot\left(\frac{\alpha^{\prime}}{1-\alpha^{\prime}}\right)^{n} \text { with } \gamma=\gamma\left(1-\alpha^{\prime}, \frac{\sigma}{2}\right),
$$

so that

(9) $\left|B_{n}\right| \leqq K^{\prime} \cdot\left\{\frac{1-\alpha}{1-\alpha^{\prime}} \cdot \frac{\alpha^{\prime}}{\alpha}\right\}^{n} \cdot e^{-\gamma^{n}}$ with $\gamma=\gamma\left(1-\alpha^{\prime}, \frac{\sigma}{2}\right)$.

Now one can easily verify that $\gamma\left(1-\alpha^{\prime}, \sigma / 2\right)$ can be taken as a continuous function of $\alpha^{\prime}$ in $0<\alpha^{\prime}<1$, if $\sigma>0$ is fixed. For $\alpha^{\prime} \rightarrow \alpha$ it tends therefore to $\gamma(1-\alpha, \sigma / 2)>0$, while the content of the braces in (9) tends to 1 . Hence, if our $\delta$ was in addition to (8) small enough (depending on $\alpha$ and on $\sigma$ and thus on $\alpha$ and on $\vartheta$ ), we have

$$
\left|B_{n}\right| \leqq K^{\prime \prime} \cdot e^{-\lambda_{1} n} \text { for some fixed constants } K^{\prime \prime}>0 \text { and } \lambda_{1}>0 ;
$$

this holds for every $n$ in the intervals $\left(n_{k}, N_{k}\right)$.

To bring (7) into a more suitable form, we notice that, by (iii), $s_{m_{k}}=O\left((1+\delta)^{m k}\right)$, and hence

$$
\begin{aligned}
s_{m_{k}} \cdot O\left(e^{-\gamma n}\right) & =O\left((1+\delta)^{m_{k}} e^{-\gamma n}\right)=O\left((1+\delta)^{n /(1-\alpha)} \cdot e^{-\gamma n}\right) \\
& =O\left(e^{-\lambda_{2} n}\right),
\end{aligned}
$$

if $\delta$ in (iii) was given so that $\log (1+\delta)<(1-\alpha) \cdot \gamma(1-\alpha, \sigma)$. Combining (6), (7), (10), (11), we obtain

$$
\begin{array}{ll}
s_{n}^{\prime}=s_{m_{k}}+O\left(e^{-\lambda n}\right) & \text { for a constant } \lambda>0 \text { and for all } \\
& n \text { in the intervals }\left(n_{k}, N_{k}\right) .
\end{array}
$$

Now we come to the easier part (b) of our program. Denoting the $C_{1}$-means of $\left\{s_{n}^{\prime}\right\}$ by $\sigma_{n}^{\prime}$, we simply write 


$$
\begin{aligned}
\sigma_{N_{k}}^{\prime} & =\frac{s_{0}^{\prime}+\cdots+s_{N_{k}}^{\prime}}{N_{k}+1}=\frac{s_{0}^{\prime}+\cdots+s_{n_{k}}^{\prime}}{N_{k}+1}+\frac{s_{n_{k}+1}^{\prime}+\cdots+s_{N_{k}}^{\prime}}{N_{k}+1} \\
& =\frac{n_{k}+1}{N_{k}+1} \sigma_{n_{k}}^{\prime}+\frac{N_{k}-n_{k}}{N_{k}+1} s_{m_{k}}+O\left(e^{-\lambda n_{k}}\right) .
\end{aligned}
$$

Assuming without loss of generality that $\sigma_{n}^{\prime} \rightarrow 0(n \rightarrow \infty)$ and remembering (5), we obtain immediately $s_{m_{k}} \rightarrow 0(k \rightarrow \infty)$, as was to be proved.

In order to prove the extension, replace the simple device (13) by the gap theorem for $C_{\boldsymbol{k}}$-summable series (see [7, p. 469]; for a simpler proof see [6] or [3]); this gap theorem is applicable to the series $\sum\left(s_{n}^{\prime}-s_{n-1}^{\prime}\right)$ because of (12), and it yields the convergence of $\left\{s_{n_{k}}^{\prime}\right\}$ and hence the convergence of $\left\{s_{m_{k}}\right\}$. Thus our extension is also proved.

\section{REFERENCES}

1. M. A. Evgrafov, On an inverse of Abel's theorem for series having gaps, Izvestiya Akademii Nauk SSSR. Ser. Mat. vol. 16 (1952) pp. 521-524.

2. D. Gaier, Über die Summierbarkeit beschränkter und stetiger Potenzreihen an der Konvergenzgrenze, Math. Zeit. vol. 56 (1952) pp. 326-334.

3. D. Gaier and K. Zeller, Über den O-Umkehrsatz fïr das $C_{k}$-Verfahren, Rend. Circ. Mat. Palermo (2) vol. 3 (1954) pp. 83-88.

4. D. Gaier and W. Meyer-König, Singuläre Radien bei Potenzreihen, Jber. Deutschen Math. Verein. vol. 59 (1956) pp. 36-48.

5. G. H. Hardy, Divergent series, Oxford, 1949.

6. H. D. Kloosterman, Limitierungsumkehrsätze mit Lïckenbedingungen für das C-Verfahren, Math. Zeit. vol. 46 (1940) pp. 375-379.

7. W. Meyer-König, Limitierungsumkehrsätze mit Lïckenbedingungen I, Math. Zeit. vol. 45 (1939) pp. 447-478.

8. M. E. Noble, On Taylor series with gaps, J. London Math. Soc. vol. 28 (1953) pp. 197-203.

9. - A further note on Taylor series with gaps, J. London Math. Soc. vol. 30 (1955) pp. 220-228.

Technische Hochschule, Stuttgart 